\title{
Existence of Strong Coupled Fixed Points for Cyclic Coupled Ciric-Type Mappings
}

\author{
Xavier Udo-utun \\ Department of Mathematics and Statistics, Faculty of Science, University of Uyo, Uyo, Nigeria \\ Correspondence should be addressed to Xavier Udo-utun; xvior@yahoo.com
}

Received 28 July 2014; Accepted 16 October 2014; Published 23 November 2014

Academic Editor: Gabriel Nagy

Copyright (C) 2014 Xavier Udo-utun. This is an open access article distributed under the Creative Commons Attribution License, which permits unrestricted use, distribution, and reproduction in any medium, provided the original work is properly cited.

In this short communication the concept of cyclic coupled Kannan-type contractions is generalized using a certain class of Cirictype mappings.

\section{Introduction and Preliminaries}

The Banach contraction condition in a metric space $(X, d)$ given by $d(T x, T y) \leq L d(x, y), 0 \leq L<1$, has so many significant generalizations which include the class of generalized contractions defined by Ciric [1] as follows. A mapping $T: X \rightarrow X$ is called a generalized contraction if and only if there exist nonnegative numbers $q$. $r, s$, and $t$ such that

$$
\begin{aligned}
d(T x, T y) \leq & q d(x, y)+r d(x, T x)+s d(y, T y) \\
& +t[d(x, T y)+d(y, T x)] \\
& \sup \{q+r+s+2 s\}<1 .
\end{aligned}
$$

$T$ is called contractive if

$$
d(T x, T y)<d(x, y) .
$$

It is worth mentioning that the contractive condition (2) restricts applications only to the class of continuous operators while the contractive conditions (1) accommodate discontinuous operators as well. The search for contractive conditions that do not require continuity of operators culminated in 1969 with the appearance of the Kannan [2] contractive condition below:

$$
d(T x, T y) \leq a[d(x, T x)+d(y, T y)], \quad 0 \leq a<\frac{1}{2} .
$$

The Chatterjea [3] contractive condition which followed is independent of both the contractive condition (2) and the
Kannan condition (3) which in turn is independent of (2). Consequently, unlike condition (2) the Kannan condition (3) does not generalize the well-known Banach condition above. In a first attempt, the three contractive conditions were combined by Zamfirescu [4] in one theorem to generalize and extend the Banach fixed point theorem. Following Zamfirescu Ciric unified contractive conditions mentioned above by introducing the larger and unifying class of operators called quasi-contractions. $T$ is called a quasi-contraction (Ćirić [5]) if there exists $L \in(0,1)$ such that

$$
\begin{gathered}
d(T x, T y) \leq L \max [d(x, y), d(x, T x), d(y, T y), \\
d(x, T y), d(y, T x)] .
\end{gathered}
$$

Ćirić [5] observed that the class of quasi-contractions contains the class of generalized contractions as a proper subclass. Rhoades [6] noted that the Zamfirescu result is generalized by the Ciric contractive condition (4).

There have been numerous generalizations and extensions of the Banach fixed point theorem in literature and they are, basically, modifications of those mentioned above. Very recently Choudhury and Maity [7] introduced the concept of cyclic coupled Kannan-type contractions and established a strong cyclic coupled fixed point result below. We recall the following definition. Let $A$ and $B$ be two nonempty subsets of a given set $X$. A mapping $F: X \times X \rightarrow X$, such that $F(x, y) \in B$ if $x \in A$ and $y \in B$ and $F(x, y) \in A$ if $x \in B$ and $y \in A$, is called a cyclic mapping with respect to $A$ and $B$. 
Definition 1 (see [8]). Let $(X, d)$ be a metric space and $A, B \subset$ $X$ nonempty subsets. $T: A \cup B \rightarrow A \cup B$ is called a cyclic (or 2-cyclic) $q$-contraction if $T A \subset B$ and $T B \subset A$ and the following condition is satisfied:

$$
d(T x, T y) \leq q d(x, y)+(1-q) \operatorname{dist}(A, B)
$$

for all $x \in A, y \in B$.

Definition 2 (see [7]). Let $A$ and $B$ be two nonempty subsets of a metric space $(X, d)$. A mapping $F: X \times X \rightarrow X$ is called a cyclic coupled Kannan-type contraction with respect to $A$ and $B$ if $F$ is cyclic with respect to $A$ and $B$ and, for some $k \in(0,1 / 2), F$ satisfies the following condition:

$$
d(F(x, y), F(u, v)) \leq k[d(x, F(x, y))+d(u, F(u, v))],
$$

where $x, v \in A$ and $y, u \in B$.

Definition 3 (see [7]). Let $X$ be a nonempty set. An element $\left(p_{x}, p_{y}\right) \in X \times X$ is called a coupled fixed point of a mapping $F: X \times X \rightarrow X$ if $p_{x}=F\left(p_{x}, p_{y}\right)$ and $p_{y}=F\left(p_{y}, p_{x}\right) . \mathrm{A}$ point $(p, p) \in X \times X$ is called a strong coupled fixed point if $F(p, p)=p$.

Theorem 4 (see [7]). Let $A$ and $B$ be two nonempty closed subsets of a complete metric space $(X, d), F: X \times X \rightarrow X$ a cyclic coupled Kannan contraction with respect to $A$ and $B$ with $A \cap B \neq \emptyset$. Then $F$ has a strong coupled fixed point in $A \cap B$.

The aim of this paper is to obtain a generalization of Theorem 4 in the context of Ciric-type contractions.

\section{Main Results}

Following Definition 2 we formulate the following definition.

Definition 5. Let $A$ and $B$ be two nonempty subsets of a metric space $(X, d)$. A mapping $F: X \times X \rightarrow X$ is called a cyclic coupled Ciric-type mapping with respect to $A$ and $B$ if $F$ is cyclic with respect to $A$ and $B$ and, for some constants $q \in(0,1), F$ satisfies the following condition:

$$
d(F(x, y), F(u, v)) \leq q M(x, u),
$$

where $x, v \in A$ and $y, u \in B$ and $M(x, u)$ is given by

$$
\begin{gathered}
M(x, u)=\max \left\{d(x, u), \frac{1}{2} d(u, F(x, y)), \frac{1}{2} d(x, F(u, v)),\right. \\
\left.\frac{1}{2}[d(x, F(x, y))+d(u, F(u, v))]\right\} .
\end{gathered}
$$

Examples of coupled cyclic Ciric-type mappings include coupled cyclic Kannan-type mappings studied in [7] since the later imply the former. We now prove the main theorem of this work.
Theorem 6. Let $A$ and $B$ be two nonempty closed subsets of $a$ complete metric space $(X, d), F: X \times X \rightarrow X$ a cyclic coupled Kannan contraction with respect to $A$ and $B$ with $A \cap B \neq \emptyset$. Then $F$ has a strong coupled fixed point in $A \cap B$.

Proof. Let $x_{0} \in A$ and $y_{0} \in B$; we put $x_{1}=F\left(y_{0}, x_{0}\right)$, $y_{1}=F\left(x_{0}, y_{0}\right), x_{2}=F\left(y_{1}, x_{1}\right), y_{2}=F\left(x_{1}, x_{1}\right)$, and so forth. Then we obtain the following as estimates for respective displacements:

$$
\begin{aligned}
& d\left(x_{1}, y_{1}\right)= d\left(F\left(y_{0}, x_{0}\right), F\left(x_{0}, y_{0}\right)\right) \\
& \leq q \max \{\left\{\left(y_{0}, x_{0}\right), \frac{1}{2} d\left(y_{0}, F\left(x_{0}, y_{0}\right)\right),\right. \\
& \frac{1}{2} d\left(x_{0}, F\left(y_{0}, x_{0}\right)\right), \\
&\left.\frac{1}{2}\left[d\left(y_{0}, F\left(y_{0}, x_{0}\right)\right)+d\left(x_{0}, F\left(x_{0}, y_{0}\right)\right)\right]\right\} \\
& \leq q \max \left\{d\left(x_{0}, y_{0}\right), \frac{1}{2} d\left(y_{0}, y_{1}\right), \frac{1}{2} d\left(x_{0}, x_{1}\right),\right.\left.\frac{1}{2}\left[d\left(y_{0}, x_{1}\right)+d\left(x_{0}, y_{1}\right)\right]\right\} \\
& \leq q \max \left\{d\left(x_{0}, y_{0}\right), d\left(x_{0}, x_{1}\right), d\left(y_{0}, y_{1}\right),\right. \\
&\left.d\left(y_{0}, x_{1}\right), d\left(x_{0}, y_{1}\right)\right\}=q \tau,
\end{aligned}
$$

where $\tau=\max \left\{d\left(x_{0}, y_{0}\right), d\left(x_{0}, x_{1}\right), d\left(y_{0}, y_{1}\right), d\left(y_{0}, x_{1}\right), d\left(x_{0}\right.\right.$, $\left.\left.y_{1}\right)\right\}$.

Similarly, using the fact that $d\left(y_{1}, x_{2}\right) \not \leq q d\left(y_{1}, x_{2}\right)$ we obtain

$$
\begin{aligned}
& d\left(y_{1}, x_{2}\right)= d\left(F\left(x_{0}, y_{0}\right), F\left(y_{1}, x_{1}\right)\right) \\
& \leq q \max \left\{d\left(x_{0}, y_{1}\right), \frac{1}{2} d\left(y_{1}, F\left(x_{0}, y_{0}\right)\right)\right. \frac{1}{2} d\left(x_{0}, F\left(y_{1}, x_{1}\right)\right), \\
& \frac{1}{2}\left[d\left(x_{0}, F\left(x_{0}, y_{0}\right)\right)\right. \\
&\left.\left.+d\left(y_{1}, F\left(y_{1}, x_{1}\right)\right)\right]\right\} \\
& \leq q \max \left\{d\left(x_{0}, y_{1}\right), \frac{1}{2} d\left(x_{0}, x_{2}\right),\right.\left.\frac{1}{2}\left[d\left(x_{0}, y_{1}\right)+d\left(y_{1}, x_{2}\right)\right]\right\} \\
& \leq q \max \left\{d\left(x_{0}, y_{1}\right), \frac{1}{2}\left[d\left(x_{0}, y_{1}\right)+d\left(y_{1}, x_{2}\right)\right]\right\}
\end{aligned}
$$

yielding $d\left(y_{1}, x_{2}\right) \leq q d\left(x_{0}, y_{1}\right) \leq q \tau$,

$$
d\left(x_{1}, y_{2}\right) \leq q d\left(y_{0}, x_{1}\right) \leq q \tau \text {. }
$$


Next, we will use (9), (11), and (12) to obtain the following estimates for the displacements $d\left(x_{1}, x_{2}\right)$ and $d\left(y_{1}, y_{2}\right)$ :

$$
\begin{aligned}
d\left(x_{1}, x_{2}\right)= & d\left(F\left(y_{0}, x_{0}\right), F\left(y_{1}, x_{1}\right)\right) \\
\leq & q \max \left\{d\left(y_{0}, y_{1}\right), \frac{1}{2} d\left(y_{1}, F\left(x_{0}, y_{0}\right)\right),\right. \\
& \frac{1}{2} d\left(y_{0}, F\left(y_{1}, x_{1}\right)\right), \\
& \left.\frac{1}{2}\left[d\left(y_{0}, F\left(x_{0}, y_{0}\right)\right)+d\left(y_{1}, F\left(y_{1}, x_{1}\right)\right)\right]\right\} \\
\leq q \max \left\{d\left(y_{0}, y_{1}\right), \frac{1}{2} d\left(y_{0}, x_{2}\right),\right. & \left.\frac{1}{2}\left[d\left(y_{0}, y_{1}\right)+d\left(y_{1}, x_{2}\right)\right]\right\} \\
\leq & q \max \left\{d\left(y_{0}, y_{1}\right), \frac{1}{2}\left[d\left(y_{0}, y_{1}\right)+d\left(y_{1}, x_{2}\right)\right],\right. \\
& \left.\frac{1}{2}\left[d\left(y_{0}, y_{1}\right)+q d\left(x_{0}, y_{1}\right)\right]\right\} \\
\leq & q \max \left\{d\left(y_{0}, y_{1}\right), \frac{1}{2}\left[d\left(y_{0}, y_{1}\right)+q d\left(x_{0}, y_{1}\right)\right]\right\}
\end{aligned}
$$

Yielding $d\left(x_{1}, x_{2}\right) \leq q \max \left\{d\left(y_{0}, y_{1}\right), q d\left(x_{0}, y_{1}\right)\right\} \leq q \tau$,

$$
d\left(y_{1}, y_{2}\right) \leq q \max \left\{d\left(x_{0}, x_{1}\right), q d\left(y_{0}, x_{1}\right)\right\} \leq q \tau .
$$

Similarly,

$$
\begin{aligned}
& d\left(y_{2}, x_{3}\right) \leq d\left(F\left(x_{1}, y_{1}\right), F\left(y_{2}, x_{2}\right)\right) \\
& \leq q \max \{ d\left(x_{1}, y_{2}\right), \frac{1}{2} d\left(y_{2}, F\left(x_{1}, y_{1}\right)\right), \\
& \frac{1}{2} d\left(x_{1}, F\left(y_{2}, x_{2}\right)\right), \\
&\left.\frac{1}{2}\left[d\left(x_{1}, F\left(x_{1}, y_{1}\right)\right)+d\left(y_{2}, F\left(y_{2}, x_{2}\right)\right)\right]\right\} \\
& \leq q \max \left\{d\left(x_{1}, y_{2}\right), \frac{1}{2} d\left(x_{1}, y_{3}\right),\right. \\
&\left.\frac{1}{2}\left[d\left(x_{1}, y_{2}\right)+d\left(y_{2}, x_{3}\right)\right]\right\} ;
\end{aligned}
$$

that is, $d\left(y_{2}, x_{3}\right) \leq q d\left(x_{1}, y_{2}\right) \leq q^{2} \tau, \quad$ by $(11)$,

$$
d\left(x_{2}, y_{3}\right) \leq q d\left(y_{1}, x_{2}\right) \leq q^{2} \tau, \quad \text { by (12). }
$$

Now, to estimate $d\left(x_{n}, x_{m}\right)$ and $d\left(x_{n}, y_{n}\right)$ we proceed as follows: given that $d\left(x_{1}, x_{2}\right) \leq q \tau, d\left(y_{1}, y_{2}\right) \leq q \tau$, $d\left(y_{2}, x_{3}\right) \leq q d\left(x_{1}, y_{2}\right) \leq q^{2} \tau$, and $d\left(x_{2}, y_{3}\right) \leq q d\left(y_{1}, x_{2}\right) \leq$ $q^{2} \tau$, we have the following:

$$
\begin{aligned}
& d\left(x_{2}, x_{3}\right)= d\left(F\left(y_{1}, x_{1}\right), F\left(y_{2}, x_{2}\right)\right) \\
& \leq q \max \left\{d\left(y_{1}, y_{2}\right), \frac{1}{2} d\left(y_{2}, F\left(y_{1}, x_{1}\right)\right),\right. \\
& \frac{1}{2} d\left(y_{1}, F\left(y_{2}, x_{2}\right)\right), \\
& \frac{1}{2}\left[d\left(y_{1}, F\left(y_{1}, x_{1}\right)\right)\right. \\
&+\left.\left.d\left(y_{2}, F\left(y_{2}, x_{2}\right)\right)\right]\right\} \\
& \leq q \max \{ d\left(y_{1}, y_{2}\right), \frac{1}{2} d\left(y_{2}, x_{2}\right), \frac{1}{2} d\left(y_{1}, x_{3}\right), \\
&\left.\frac{1}{2}\left[d\left(y_{1}, x_{2}\right)+d\left(y_{2}, x_{3}\right)\right]\right\}, \quad \text { by }(14)
\end{aligned}
$$$$
\text { yielding } d\left(x_{2}, x_{3}\right) \leq q \max \left\{q \tau, q^{2} \tau\right\}=q^{2} \tau \text {, }
$$$$
d\left(y_{2}, y_{3}\right) \leq q^{2} \tau, \quad \text { by }(15) \text {. }
$$

Inductively, we assume $d\left(x_{k}, x_{k+1}\right) \leq q^{k} \tau$ and $d\left(y_{k}, y_{k+1}\right) \leq$ $q^{k} \tau ; k \geq 1$. Also, we assume $d\left(x_{k}, y_{k+1}\right) \leq q^{k} \tau$ and $d\left(y_{k}, x_{k+1}\right) \leq q^{k} \tau ; k \geq 1$. Then

$$
d\left(y_{k+1}, x_{k+2}\right)=d\left(F\left(x_{k}, y_{k}\right), F\left(y_{k+1}, x_{k+1}\right)\right)
$$

$$
\begin{gathered}
\leq q \max \left\{d\left(x_{k}, y_{k+1}\right), \frac{1}{2} d\left(y_{k+1}, F\left(x_{k}, y_{k}\right)\right),\right. \\
\frac{1}{2} d\left(x_{k}, F\left(y_{k+1}, x_{k+1}\right)\right), \\
\frac{1}{2}\left[d\left(x_{k}, F\left(x_{k}, y_{k}\right)\right)\right. \\
\left.\left.+d\left(y_{k+1}, F\left(y_{k+1}, x_{k+1}\right)\right)\right]\right\} \\
\leq q \max \left\{d\left(x_{k}, y_{k+1}\right), \frac{1}{2} d\left(x_{k}, x_{k+2}\right),\right. \\
\left.\frac{1}{2}\left[d\left(x_{k}, y_{k+1}\right)+d\left(y_{k+1}, x_{k+2}\right)\right]\right\} \\
\leq q \max \left\{d\left(x_{k}, y_{k+1}\right),\right. \\
\left.\frac{1}{2}\left[d\left(x_{k}, y_{k+1}\right)+d\left(y_{k+1}, x_{k+2}\right)\right]\right\}
\end{gathered}
$$

yielding $d\left(y_{k+1}, x_{k+2}\right) \leq q d\left(x_{k}, y_{k+1}\right) \leq q^{k+1} \tau$,

$$
d\left(x_{k+1}, y_{k+2}\right) \leq q d\left(y_{k}, x_{k+1}\right) \leq q^{k+1} \tau .
$$


It follows from (19) and (20) that

$$
\begin{aligned}
& d\left(x_{k+1}, x_{k+2}\right)= d\left(F\left(y_{k}, x_{k}\right), F\left(y_{k+1}, x_{k+1}\right)\right) \\
& \leq q \max \{ d\left(y_{k}, y_{k+1}\right), \frac{1}{2} d\left(y_{k+1}, F\left(x_{k}, y_{k}\right)\right), \\
& \frac{1}{2} d\left(y_{k}, F\left(y_{k+1}, x_{k+1}\right)\right), \\
& \frac{1}{2}\left[d\left(y_{k}, F\left(x_{k}, y_{k}\right)\right)\right.\left.\left.+d\left(y_{k+1}, F\left(y_{k+1}, x_{k+1}\right)\right)\right]\right\} \\
& \leq q \max \left\{d\left(y_{k}, y_{k+1}\right), \frac{1}{2} d\left(y_{k}, x_{k+2}\right),\right. \\
&\left.\frac{1}{2}\left[d\left(y_{k}, y_{k+1}\right)+d\left(y_{k+1}, x_{k+2}\right)\right]\right\} \\
& \leq q \max \left[d\left(y_{k}, y_{k+1}\right), d\left(y_{k}, x_{k+1}\right),\right. \\
&\left.d\left(y_{k+1}, x_{k+2}\right)\right]
\end{aligned}
$$

so we have $d\left(x_{k+1}, x_{k+2}\right) \leq q \max \left[q^{k} \tau, q^{k+1} \tau\right] \leq q^{k+1} \tau$,

$$
d\left(y_{k+1}, y_{k+2}\right) \leq q^{k+1} \tau
$$

Therefore, using (19)-(23) we conclude that for all $n \geq 1$ we have $d\left(x_{n}, x_{n+1}\right) \leq q^{n} \tau$ and $d\left(x_{n}, x_{n+1}\right) \leq q^{n} \tau$. Also, using the fact that $d\left(x_{n}, y_{n}\right) \leq d\left(x_{n}, y_{n+1}\right)+d\left(y_{n}, y_{n+1}\right)$ we conclude that $d\left(x_{n}, y_{n}\right) \leq q^{n} 2 \tau$ for all $n \geq 1$ implying that if $p_{x}=\lim x_{n}$ and $p_{y}=\lim y_{n}$ then $p_{y}=p_{x}=p$ and $(p, p) \in \operatorname{Fix}(F)$. To prove that the sequences $\left\{x_{n}\right\}$ and $\left\{y_{n}\right\}$ are convergent it suffices to verify that they are Cauchy sequences.

To this end, we observe that if $m \geq n$ then $m=n+l$ for some $l \geq 0$ so that

$$
\begin{aligned}
d\left(x_{n}, x_{m}\right)= & d\left(x_{n}, x_{n+p}\right) \\
\leq & d\left(x_{n}, x_{n+1}\right)+d\left(x_{n+1}, x_{n+2}\right)+\cdots \\
& +d\left(x_{m-1}, x_{n+l}\right) \\
\leq & q^{n}\left[1+q+q^{2}+\cdots+q^{l}\right] \tau \\
\leq & \frac{q^{n}}{1-q} \tau .
\end{aligned}
$$

Therefore, the sequences $\left\{x_{n}\right\}$ and $\left\{y_{n}\right\}$ are Cauchy sequences and since $A$ and $B$ are closed sets with $A \cap B \neq \emptyset$ we submit that the strong cyclic coupled fixed point $(p, p)$ of $F$ so derived satisfies $p \in A \cap B$.

\section{Conclusion}

Further studies include proving our main result and Theorem 4 without the restriction $A \cap B \neq \emptyset$. This can be studied as a best proximity point problem.

\section{Conflict of Interests}

The author declares that there is no conflict of interests regarding the publication of this paper.

\section{References}

[1] L. B. Ciric, "Generalized contractions and fixed-point theorems," Publications de l'Institut Mathématique, vol. 12, no. 26, pp. 19-26, 1971.

[2] R. Kannan, "Some result on fixed points II," The American Mathematical Monthly, vol. 76, pp. 405-406, 1969.

[3] S. K. Chatterjea, "Fixed-point theorems," Comptes Rendus de l'Academie Bulgare des Sciences, vol. 25, pp. 727-730, 1972.

[4] T. Zamfirescu, "Fix point theorems in metric spaces," Archiv der Mathematik, vol. 23, no. 1, pp. 292-298, 1972.

[5] L. B. Ćirić, "Generalzation of Banach's contraction principle," Proceedings of the American Mathematical Society, vol. 45, no. 2, pp. 267-273, 1974.

[6] B. E. Rhoades, "A comparison of various definitions of contractive mappings," Transactions of the American Mathematical Society, vol. 226, pp. 257-290, 1977.

[7] B. S. Choudhury and P. Maity, "Cyclic coupled fixed point result using Kannan type contractions," Journal of Operators, vol. 2014, Article ID 876749, 5 pages, 2014.

[8] M. De la Sen, "Linking contractive self-mappings and cyclic Meir-Keeler contractions with KANnan self-mappings," Fixed Point Theory and Applications, vol. 2010, Article ID 572057, 23 pages, 2010. 


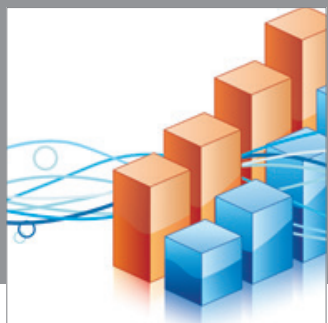

Advances in

Operations Research

mansans

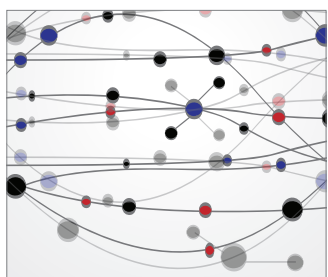

The Scientific World Journal
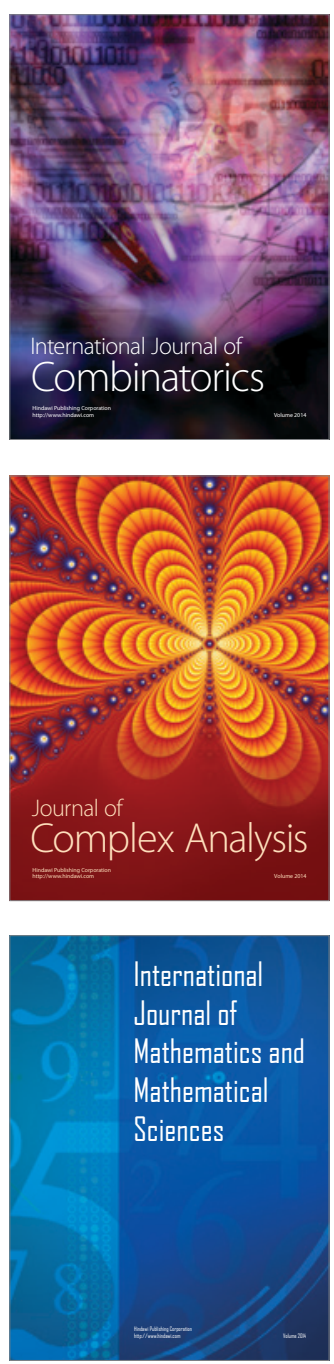
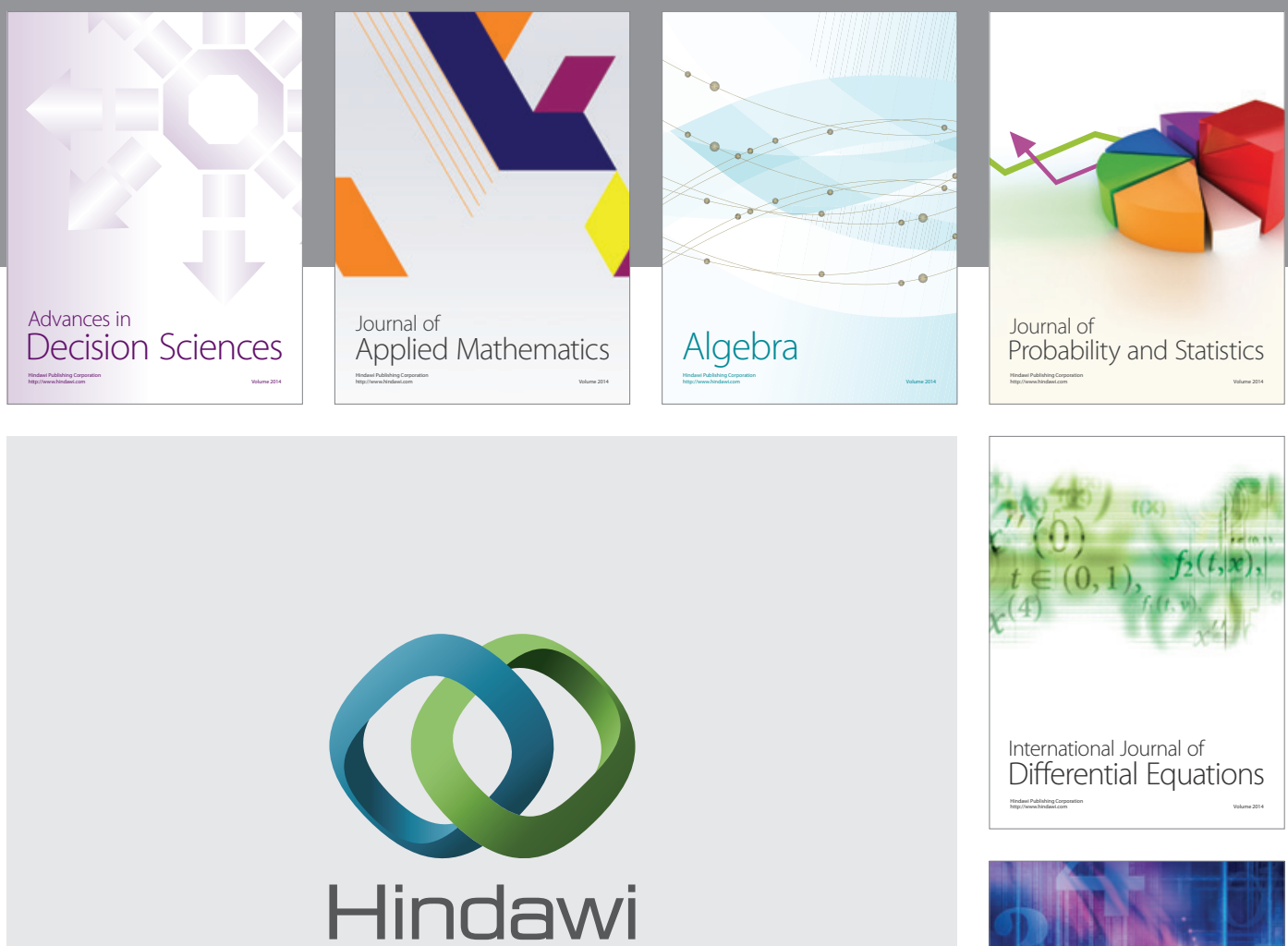

Submit your manuscripts at http://www.hindawi.com
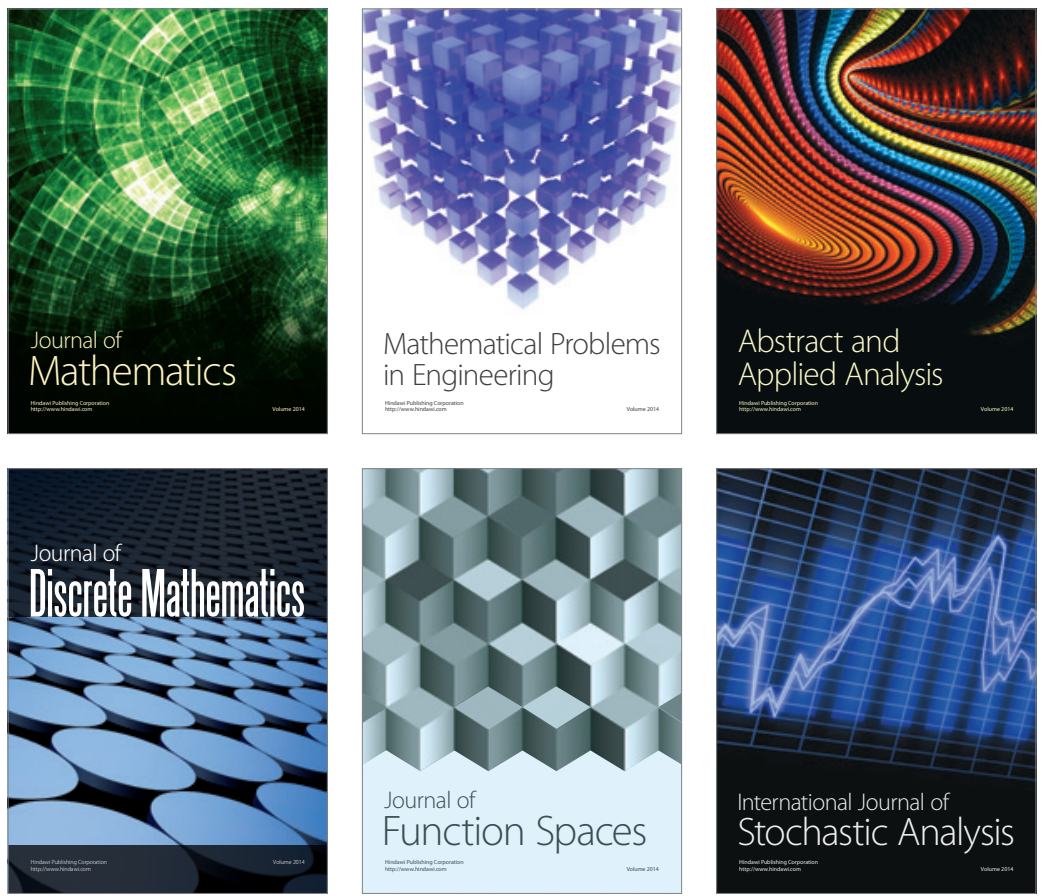

Journal of

Function Spaces

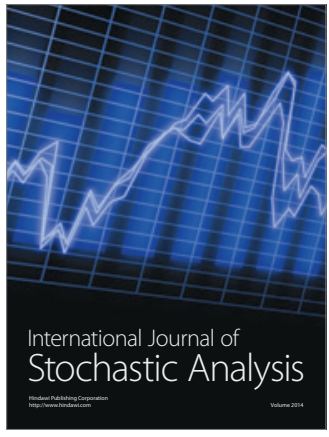

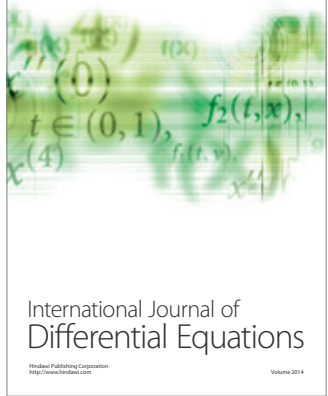
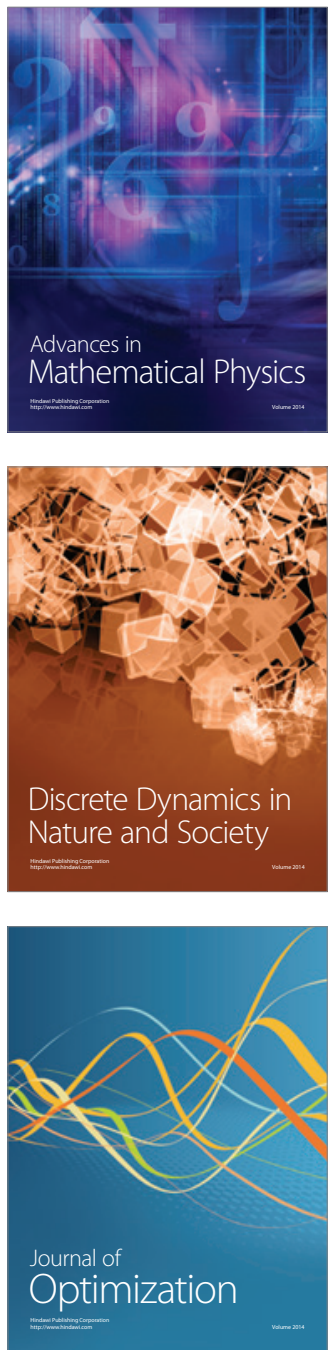